\title{
Effect of predictors of visual preference as characteristics of urban natural landscapes in increasing perceived restorative potential
}

\begin{abstract}
Current literature on restorative environments generally shows the importance of urban natural landscapes in increasing perceived restorativeness of urban environments. However, little is known about the positive role of visual characteristics of urban natural landscapes in increasing Perceived Restorative Potential (PRP) of urban environments. This study aimed to understand the role of four predictors of visual preference as characteristics of urban natural landscape on the restorativeness of environments. In this study, 120 students from Universiti Putra Malaysia rated 12 colored slides of urban natural landscape scenes for four restorative components (Being away, Fascination, Coherence, and Compatibility), four predictors of visual landscape preference (Coherence, Complexity, Legibility, Mystery) and a criterion variable (PRP). Coherence, however, was considered as a predictor of visual landscape preference since it is not a significant predictor in explaining PRP. Results indicate that Coherence, Complexity, and Mystery positively explained PRP while Legibility did not. Further analysis revealed that the effect of Coherence and Complexity on PRP was fully mediated by the restorative components and Mystery was only partially mediated. The findings indicate that some predictors of visual landscape preference of urban natural landscapes contribute to increasing PRP of urban landscapes. Although Coherence as a restorative component was not a significant predictor of PRP, it contributed toward increasing the restorative potential of urban natural landscapes when considered as a predictor of visual landscape preference. The results of this study can aid city planners, landscape architects and developers with regards to the influence of visual landscape characteristics in increasing the restorative potential of urban environments.
\end{abstract}

Keyword: Attention restoration theory; Information processing theory; Landscape design; Mental fatigue; Restorative environment 